\title{
The on-shell massless planar double box diagram with an irreducible numerator
}

\author{
C. Anastasiou, ${ }^{a *}$ J.B. Tausk ${ }^{\mathrm{b} \dagger}$ and M.E. Tejeda-Yeomans ${ }^{\mathrm{a} \ddagger}$ \\ a Department of Physics, University of Durham, Durham DH1 3LE, England \\ b Fakultät für Physik, Albert-Ludwigs-Universität Freiburg, D-79104 Freiburg, Germany
}

Using a Mellin-Barnes representation, we compute the on-shell massless planar double box Feynman diagram with an irreducible scalar product of loop momenta in the numerator. This diagram is needed in calculations of two loop corrections to scattering processes of massless particles, together with the double box without numerator calculated previously by Smirnov. We verify the poles in $\epsilon$ of our result by means of a system of differential equations relating the two diagrams, which we present in an explicit form. We verify the finite part with an independent numerical check.

\section{Introduction}

We shall consider the following class of Feynman integrals

$$
\begin{gathered}
I\left(\nu_{1}, \nu_{2}, \nu_{3}, \nu_{4}, \nu_{5}, \nu_{6}, \nu_{7}, \nu_{8} ; d\right)=\iint \mathrm{d}^{d} k \mathrm{~d}^{d} l \\
\frac{1}{P_{1}^{\nu_{1}} P_{2}^{\nu_{2}} P_{3}^{\nu_{3}} P_{4}^{\nu_{4}} P_{5}^{\nu_{5}} P_{6}^{\nu_{6}} P_{7}^{\nu_{7}} P_{8}^{\nu_{8}}}
\end{gathered}
$$

where the propagator denominators $P_{i}$ are defined by $P_{1}=k^{2}, P_{2}=\left(k+p_{1}\right)^{2}, P_{3}=\left(k+p_{1}+\right.$ $\left.p_{2}\right)^{2}, P_{4}=\left(l+p_{1}+p_{2}\right)^{2}, P_{5}=\left(l+p_{1}+p_{2}+p_{3}\right)^{2}$, $P_{6}=l^{2}, P_{7}=(l-k)^{2}, P_{8}=\left(k+p_{1}+p_{2}+p_{3}\right)^{2}$, $d=4-2 \epsilon$ is the space-time dimension, and the external momenta $p_{1}, p_{2}, p_{3}$, and $p_{4}=-p_{1}-p_{2}-p_{3}$ are lightlike. The integrals (11) then depend on two scales: $s=\left(p_{1}+p_{2}\right)^{2}$ and $t=\left(p_{2}+p_{3}\right)^{2}$. We shall mainly be concerned with cases where $\nu_{8}$ is either zero or a negative integer. These integrals correspond to the diagram shown in figure 1, and are important for calculations of two-loop scattering amplitudes of massless particles, for example in QCD [1, 2].

The scalar double box integral

$$
I_{1}^{d}(s, t)=I(1,1,1,1,1,1,1,0 ; d),
$$

was calculated analytically by Smirnov [ [3] as a Laurent expansion in $\epsilon$ to $\mathcal{O}\left(\epsilon^{0}\right)$. Subsequently,

\footnotetext{
${ }^{*}$ Ch.Anastasiou@durham.ac.uk

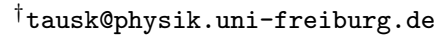

${ }^{\ddagger}$ M.E.Tejeda-Yeomans@durham.ac.uk
}

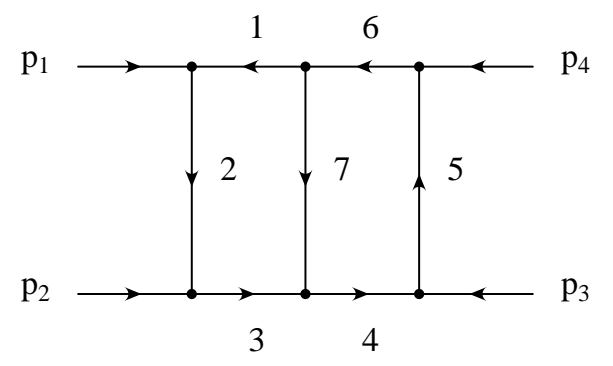

Figure 1. The planar double box diagram

Smirnov and Veretin constructed a general algorithm [ [ grals. In this algorithm, integrals with arbitrary polynomials of loop momenta in the numerator are first expressed in terms of scalar integrals $I\left(\nu_{1}, \nu_{2}, \nu_{3}, \nu_{4}, \nu_{5}, \nu_{6}, \nu_{7}, 0 ; d+2 n\right)$ with increased integer powers of the propagators in shifted spacetime dimensions [5]. Then, using integration by parts identities [6], these scalar integrals are systematically reduced to linear combinations of a set of master integrals, consisting of $I_{1}$, a second master double box integral,

$I_{2}^{d}(s, t)=I(1,1,1,1,1,1,2,0 ; d)$,

and a small number of more simple master inte- 
grals (see figure 2). Furthermore, they gave an explicit analytical expression for the second master integral $I_{2}$, again as a Laurent expansion in $\epsilon$ to $\mathcal{O}\left(\epsilon^{0}\right)$.

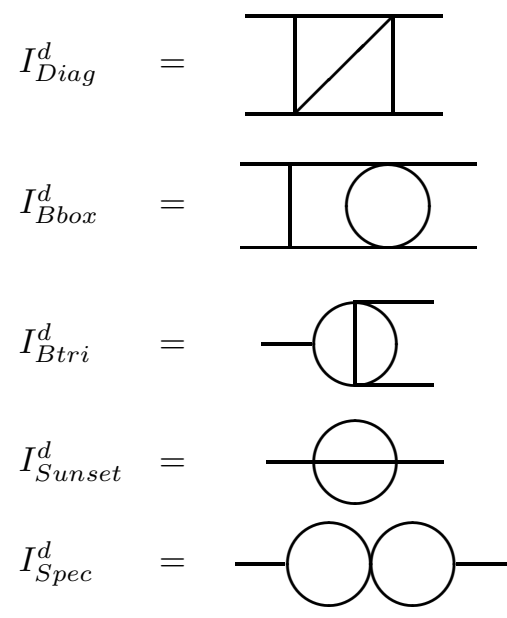

Figure 2. Master integrals with less than six propagators

It appeared that the algorithm described in ref. [4] completely solved the problem of calculating on-shell double box diagrams. However, as was reported by Glover at this workshop [2], it often happens that in the reduction of a given tensor integral, the coefficients in front of the master integrals $I_{1}$ and $I_{2}$ are of order $\mathcal{O}(1 / \epsilon)$. This is a consequence of the fact that in the reduction of these integrals it is necessary to reduce the dimension down to $d=4-2 \epsilon$ from at least $d=6-2 \epsilon$, and in the system of equations for the dimensional shift, there are factors of $1 /(d-6)$ sitting in front of $I_{1}$ and $I_{2}$. Thus, in order to calculate such tensor integrals to $\mathcal{O}(\epsilon)$, one would need to know $I_{1}$ and $I_{2}$ to one order higher in $\epsilon$ than they are given in refs. [3, 4.

A typical example is the following integral with an irreducible numerator:

$I_{I r r}^{d}(s, t)=I(1,1,1,1,1,1,1,-1 ; d)$, whose reduction to master integrals reads [2]:

$$
\begin{aligned}
I_{\text {Irr }}^{d}( & s, t)=-\frac{1}{2} \frac{(3 d-14) s}{d-4} I_{1}^{d}(s, t) \\
& -\frac{1}{2} \frac{(d-6) s t}{(d-4)(d-5)} I_{2}^{d}(s, t) \\
& -3 \frac{(s+t)}{(d-5)(d-6) s^{2} t} \\
& \times\left[\left(7 d^{2}-68 d+164\right) s\right. \\
& +(3 d-14)(3 d-16) t] I_{\text {Diag }}^{d}(s, t) \\
& +24 \frac{(d-3)}{(d-6) t} I_{B b o x}^{d}(s, t) \\
& -4 \frac{(d-3)^{2}(2 d-9)}{(d-4)^{2}(d-5) s^{2}} I_{\text {Spec }}^{d}(s) \\
& +\frac{3}{2} \frac{(d-3)(3 d-10)}{(d-4)^{2}(d-5)^{2}(d-6) s^{2} t} \\
& \times\left[8(d-4)(d-5)^{2} s\right. \\
& \left.+\left(-11 d^{3}+158 d^{2}-754 d+1196\right) t\right] I_{\text {Btri }}^{d}(s) \\
& +3 \frac{(d-3)(3 d-8)(3 d-10)}{(d-4)^{3}(d-5)^{2}(d-6) s^{3} t} \\
& \times\left[(d-5)\left(7 d^{2}-68 d+164\right) s\right. \\
& \left.+\left(23 d^{3}-337 d^{2}+1640 d-2652\right) t\right] I_{\text {Sunset }}^{d}(s) \\
+ & 3 \frac{(d-3)(3 d-8)(3 d-10)}{(d-4)^{3}(d-5)^{2}(d-6) s^{2} t^{2}} \\
& +\left(d-5 d^{3}-229 d^{2}+1088 d-1716\right) s \\
& \\
& \\
&
\end{aligned}
$$

where

$$
\begin{aligned}
I_{\text {Diag }}^{d}(s, t) & =I(0,1,1,0,1,1,1,0 ; d), \\
I_{\text {Bbox }}^{d}(s, t) & =I(1,1,1,0,1,0,1,0 ; d), \\
I_{\text {Spec }}^{d}(s) & =I(1,0,1,1,0,1,0,0 ; d), \\
I_{\text {Sunset }}^{d}(s) & =I(0,0,1,0,0,1,1,0 ; d), \\
I_{\text {Sunset }}^{d}(t) & =I(0,1,0,0,1,0,1,0 ; d), \\
I_{\text {Btri }}^{d}(s) & =I(1,0,1,0,1,0,1,0 ; d) .
\end{aligned}
$$

The factors of $(d-4)$ in the denominators of the first two terms on the right hand side of eq. (5) are the ones we are concerned about.

The purpose of this paper is to calculate the integral with the irreducible numerator $I_{\text {Irr }}$ directly from a Mellin-Barnes representation. It can then be used instead of $I_{2}$ as a new master integral. We 
check our result in two different ways: firstly, by verifying that $I_{1}$ and $I_{I r r}$ satisfy a system of differential equations, and secondly, by using them to compute $I_{1}$ and $I_{2}$ in $d=6$ dimensions, both of which are finite, and comparing the result with a numerical integration.

\section{Calculation by Mellin-Barnes contour integrals}

The analytic structure of the on-shell double box is rather simple, since it only depends on two scales, and its only thresholds are at $s=0$ and $t=0$. The main difficulty in calculating this diagram is to find a way to isolate its infrared and collinear divergences. A general recursive solution to this problem based on Feynman parameters was proposed in ref. [7]. However, for the analytical calculation we are going to present here, it is more convenient to use a Mellin-Barnes representation [ [3, 8], which enables us to isolate the poles in $\epsilon$ in a very natural way.

We derive our Mellin-Barnes representation for the two-loop integrals (11) by doing the loop integrations one by one (cf. the off-shell calculation [ 9]). In terms of Feynman parameters, the $l$-loop can be written as

$$
\begin{aligned}
\int \mathrm{d}^{d} l & \frac{1}{P_{4}^{\nu_{4}} P_{5}^{\nu_{5}} P_{6}^{\nu_{6}} P_{7}^{\nu_{7}}}=i \pi^{d / 2}(-1)^{\nu_{4567}} \\
& \times \frac{\Gamma\left(\nu_{4567}-d / 2\right)}{\Gamma\left(\nu_{4}\right) \Gamma\left(\nu_{5}\right) \Gamma\left(\nu_{6}\right) \Gamma\left(\nu_{7}\right)} \int_{0}^{\infty} \prod_{j=4}^{7} \mathrm{~d} x_{j} x_{j}^{\nu_{j}-1} \\
& \times \delta\left(1-x_{4}-x_{5}-x_{6}-x_{7}\right) C^{d / 2-\nu_{4567}}
\end{aligned}
$$

where $\nu_{4567}=\nu_{4}+\nu_{5}+\nu_{6}+\nu_{7}$ (similar abbreviations will be used below), and

$C=-P_{1} x_{6} x_{7}-P_{3} x_{4} x_{7}-P_{8} x_{5} x_{7}-s x_{4} x_{6}$.

By introducing three Mellin-Barnes parameters, $\alpha, \beta$ and $\tau$, we split the polynomial $C$ into factors:

$$
\begin{gathered}
\Gamma\left(\nu_{4567}-d / 2\right) C^{d / 2-\nu_{4567}}=\int_{-i \infty}^{i \infty} \frac{\mathrm{d} \alpha}{2 \pi i} \int_{-i \infty}^{i \infty} \frac{\mathrm{d} \beta}{2 \pi i} \\
\int_{-i \infty}^{i \infty} \frac{\mathrm{d} \tau}{2 \pi i}\left(-P_{1} x_{6} x_{7}\right)^{\alpha}\left(-P_{3} x_{4} x_{7}\right)^{\beta}\left(-P_{8} x_{5} x_{7}\right)^{\tau}
\end{gathered}
$$

$$
\begin{aligned}
& \times\left(-s x_{4} x_{6}\right)^{d / 2-\nu_{4567}-\alpha-\beta-\tau} \Gamma(-\alpha) \Gamma(-\beta) \\
& \times \Gamma(-\tau) \Gamma\left(\nu_{4567}-d / 2+\alpha+\beta+\tau\right) .
\end{aligned}
$$

After inserting eq. (9) into eq. (7), we evaluate the Feynman parameter integrals in terms of gamma functions, which gives us the following MellinBarnes representation for the $l$-loop:

$$
\begin{aligned}
& \int \mathrm{d}^{d} l \frac{1}{P_{4}^{\nu_{4}} P_{5}^{\nu_{5}} P_{6}^{\nu_{6}} P_{7}^{\nu_{7}}}=\frac{i \pi^{d / 2}(-1)^{\nu_{4567}}}{\Gamma\left(\nu_{4}\right) \Gamma\left(\nu_{5}\right) \Gamma\left(\nu_{6}\right) \Gamma\left(\nu_{7}\right)} \\
& \times \frac{1}{\Gamma\left(d-\nu_{4567}\right)} \int_{-i \infty}^{i \infty} \frac{\mathrm{d} \alpha}{2 \pi i} \int_{-i \infty}^{i \infty} \frac{\mathrm{d} \beta}{2 \pi i} \int_{-i \infty}^{i \infty} \frac{\mathrm{d} \tau}{2 \pi i} \\
& \times\left(-P_{1}\right)^{\alpha}\left(-P_{3}\right)^{\beta}\left(-P_{8}\right)^{\tau}(-s)^{d / 2-\nu_{4567}-\alpha-\beta-\tau} \\
& \times \Gamma(-\alpha) \Gamma(-\beta) \Gamma(-\tau) \Gamma\left(\nu_{4567}-d / 2+\alpha+\beta+\tau\right) \\
& \times \Gamma\left(d / 2-\nu_{567}-\alpha-\tau\right) \Gamma\left(d / 2-\nu_{457}-\beta-\tau\right) \\
& \times \Gamma\left(\nu_{5}+\tau\right) \Gamma\left(\nu_{7}+\alpha+\beta+\tau\right) .
\end{aligned}
$$

When this result is inserted into (11), the remaining $k$-integral has the form of an on-shell massless one-loop box diagram with indices $\nu_{1}-\alpha$, $\nu_{2}, \nu_{3}-\beta, \nu_{8}-\tau$. We repeat the above steps for this $k$-integral, using a further Mellin-Barnes parameter, $\sigma$, and finally obtain

$$
\begin{aligned}
& I\left(\nu_{1}, \nu_{2}, \nu_{3}, \nu_{4}, \nu_{5}, \nu_{6}, \nu_{7}, \nu_{8} ; d\right)= \\
& \frac{\left(i \pi^{d / 2}\right)^{2}(-1)^{\nu_{12345678}}}{\Gamma\left(\nu_{2}\right) \Gamma\left(\nu_{4}\right) \Gamma\left(\nu_{5}\right) \Gamma\left(\nu_{6}\right) \Gamma\left(\nu_{7}\right) \Gamma\left(d-\nu_{4567}\right)} \frac{1}{(2 \pi i)^{4}} \\
& \times \int_{-i \infty}^{i \infty} \mathrm{d} \alpha \int_{-i \infty}^{i \infty} \mathrm{d} \beta \int_{-i \infty}^{i \infty} \mathrm{d} \tau \int_{-i \infty}^{i \infty} \mathrm{d} \sigma(-t)^{-\sigma}(-s)^{d-\nu+\sigma} \\
& \times \frac{\Gamma(-\alpha) \Gamma(-\beta) \Gamma(-\tau) \Gamma\left(\nu_{4567}-d / 2+\alpha+\beta+\tau\right)}{\Gamma\left(\nu_{1}-\alpha\right) \Gamma\left(\nu_{3}-\beta\right) \Gamma\left(\nu_{8}-\tau\right)} \\
& \times \frac{\Gamma(\sigma) \Gamma\left(\nu_{1238}-d / 2-\alpha-\beta-\tau-\sigma\right)}{\Gamma\left(d-\nu_{1238}+\alpha+\beta+\tau\right)} \\
& \times \Gamma\left(d / 2-\nu_{567}-\alpha-\tau\right) \Gamma\left(d / 2-\nu_{457}-\beta-\tau\right) \\
& \times \Gamma\left(\nu_{5}+\tau\right) \Gamma\left(\nu_{7}+\alpha+\beta+\tau\right) \\
& \times \Gamma\left(d / 2-\nu_{128}+\alpha+\tau+\sigma\right) \Gamma\left(\nu_{8}-\tau-\sigma\right) \\
& \times \Gamma\left(d / 2-\nu_{238}+\beta+\tau+\sigma\right) \Gamma\left(\nu_{2}-\sigma\right) .
\end{aligned}
$$

In deriving this formula, we have assumed that the various parameters are such, that all the manipulations we performed are justified. This is certainly true if we are able to find a set of straight lines, parallel to the imaginary axis, for the inte- 
gration variables $\alpha, \beta, \sigma$, and $\tau$, such that the arguments of all the gamma functions in it have positive real parts. We then define the integrals (11) for values of the parameters where such contours do not exist by analytic continuation.

Let us now consider the case with the irreducible numerator, $I_{\text {Irr }}$. On the one hand, from the definition (何), $\nu_{5}+\nu_{8}=1-1=0$. On the other hand, if the real parts of the arguments of all gamma functions are positive, then in particular $\operatorname{Re}\left(\nu_{5}+\tau\right), \operatorname{Re}(\sigma)$ and $\operatorname{Re}\left(\nu_{8}-\tau-\sigma\right)$ are positive, and therefore $\operatorname{Re}\left(\nu_{5}+\nu_{8}\right)>0$. Since this does not depend on $d$, it means that in order to calculate $I_{\text {Irr }}$ using the Mellin-Barnes representation (11), we must perform an analytic continuation not only in $d$, but also in $\nu_{5}$ or $\nu_{8}$. We choose $\nu_{8}$. Setting $\nu_{8}=-1+\eta$ and all other $\nu$ 's equal to one, we get

$$
\begin{aligned}
& I_{\text {Irr }}=\lim _{\eta \downarrow 0} I(1,1,1,1,1,1,-1+\eta ; d)=\frac{\left(i \pi^{d / 2}\right)^{2}}{\Gamma(-2 \epsilon)} \\
& \times \lim _{\eta \downarrow 0} \frac{1}{(2 \pi i)^{4}} \int_{-i \infty}^{i \infty} \mathrm{d} \alpha \int_{-i \infty}^{i \infty} \mathrm{d} \beta \int_{-i \infty}^{i \infty} \mathrm{d} \tau \int_{-i \infty}^{i \infty} \mathrm{d} \sigma(-t)^{-\sigma} \\
& \times(-s)^{-2-\eta-2 \epsilon+\sigma} \Gamma(\sigma) \Gamma(1-\sigma) \\
& \times \frac{\Gamma(-\alpha) \Gamma(-\beta) \Gamma(-\tau) \Gamma(1+\tau) \Gamma(-1+\eta-\tau-\sigma)}{\Gamma(1-\alpha) \Gamma(1-\beta) \Gamma(-1+\eta-\tau)} \\
& \times \frac{\Gamma(1+\alpha+\beta+\tau) \Gamma(2+\epsilon+\alpha+\beta+\tau)}{\Gamma(2-\eta-2 \epsilon+\alpha+\beta+\tau)} \\
& \times \Gamma(-1-\epsilon-\alpha-\tau) \Gamma(1-\eta-\epsilon+\alpha+\tau+\sigma) \\
& \times \Gamma(-1-\epsilon-\beta-\tau) \Gamma(1-\eta-\epsilon+\beta+\tau+\sigma) \\
& \times \Gamma(\eta+\epsilon-\alpha-\beta-\tau-\sigma) .
\end{aligned}
$$

We can make the real parts of the arguments of all gamma functions in eq. 12 positive by picking, for example, $\eta=12 y$ and $\epsilon=-12 y$, where $y$ is some positive number much smaller than one, and choosing contours for the Mellin-Barnes variables defined by: $\operatorname{Re}(\alpha)=\operatorname{Re}(\beta)=-y, \operatorname{Re}(\tau)=-1+$ $4 y$ and $\operatorname{Re}(\sigma)=4 y$.

Starting from these values, we first perform an analytic continuation in $\eta$ to $\eta=0$, keeping $\epsilon$ fixed, and then another one in $\epsilon$ to the vicinity of $\epsilon=0$. The procedure for both continuations is straightforward: keeping the integration contours fixed, we simply have to keep track of the poles of the gamma functions, and whenever one of them crosses an integration contour, add its residue to a list of terms that will contribute to the final answer. For example, with the above choice of contours, the first crossing happens at $\eta=8 y$, when the pole at $\tau=-1+\eta-\sigma$ crosses the $\tau$ contour. After taking a residue in one integration variable, we continue to follow the poles in the remaining variables, building up a tree of single and multiple residue terms. By this procedure, poles in $\epsilon$ are automatically expressed through singular gamma functions multiplying integrals that can safely be expanded under the integral sign.

To $\mathcal{O}\left(\epsilon^{0}\right)$, it turns out that, along with terms where there is no integral left, only single and two-fold integrals contribute, because terms with more integrals are killed by the factor $1 / \Gamma(-2 \epsilon)$ in eq. (12). In the two-fold integrals, one integration can be done by Barnes's first lemma [10]. The single integrals that are left can all be calculated by closing the contour and summing harmonic series. We find the following result:

$$
\begin{aligned}
& I_{\text {Irr }}=\frac{\left(i \pi^{d / 2}\right)^{2} \Gamma(1+\epsilon)^{2}}{s^{2}(-s)^{2 \epsilon}}\left\{\frac{9}{4 \epsilon^{4}}-\frac{2}{\epsilon^{3}} \ell-\frac{7 \pi^{2}}{3 \epsilon^{2}}\right. \\
& +\frac{1}{\epsilon}\left[\frac{4}{3} \ell^{3}+\frac{14}{3} \pi^{2} \ell-4\left(\ell^{2}+\pi^{2}\right) \log (1+t / s)\right. \\
& \left.\quad+8 \operatorname{Li}_{3}(-t / s)-8 \ell \operatorname{Li}_{2}(-t / s)-16 \zeta(3)\right] \\
& -\frac{4}{3} \ell^{4}-\frac{13}{3} \pi^{2} \ell^{2}+\left(\frac{16}{3} \ell^{3}+\frac{26}{3} \pi^{2} \ell\right) \log (1+t / s) \\
& -5\left(\ell^{2}+\pi^{2}\right) \log ^{2}(1+t / s) \\
& +\left(6 \ell^{2}-20 \ell \log (1+t / s)-\frac{4}{3} \pi^{2}\right) \operatorname{Li}_{2}(-t / s) \\
& +(8 \ell+20 \log (1+t / s)) \operatorname{Li}_{3}(-t / s) \\
& +20 \mathrm{~S}_{2,2}(-t / s)-20 \ell \mathrm{S}_{1,2}(-t / s)-28 \operatorname{Li}_{4}(-t / s) \\
& \left.+(28 \ell-20 \log (1+t / s)) \zeta(3)-\frac{7 \pi^{4}}{45}\right\},
\end{aligned}
$$

with $\ell=\log (t / s)$.

\section{Differential equations for double-box master integrals}

The integrals $I_{1}$ and $I_{I r r}$ can now be chosen as a new basis of master integrals of the planar double-box topology, solving the problem of the 
$\mathcal{O}(1 / \epsilon)$ coefficients of $I_{1}$ and $I_{2}$ in matrix element calculations [2].

It is possible to construct a system of differential equations satisfied by the double box master integrals [ [1, 11]. In terms of the new basis, these differential equations are [2]:

$$
\begin{aligned}
& \frac{\partial}{\partial t} I_{1}^{d}(s, t)=\frac{[(d-5) s-t]}{(s+t) t} I_{1}^{d}(s, t) \\
& +\frac{(d-4)}{(s+t) t} I_{I r r}^{d}(s, t) \\
& -6 \frac{(d-4)}{s t^{2}} I_{\text {Diag }}^{d}(s, t) \\
& +12 \frac{(d-3)}{(s+t) t^{2}} I_{B b o x}^{d}(s, t) \\
& +4 \frac{(d-3)^{2}}{(d-4) s^{2} t(s+t)} I_{\text {Spec }}^{d}(s) \\
& +3 \frac{(d-3)(3 d-10)(2 s+t)}{(d-4) s^{2} t^{2}(s+t)} I_{B t r i}^{d}(s) \\
& +6 \frac{(d-3)(3 d-8)(3 d-10)(s-t)}{(d-4)^{2} s^{3} t^{2}(s+t)} I_{\text {Sunset }}^{d}(s) \\
& +6 \frac{(d-3)(3 d-8)(3 d-10)}{(d-4)^{2} s t^{3}(s+t)} I_{\text {Sunset }}^{d}(t) \\
& \frac{\partial}{\partial t} I_{I r r}^{d}(s, t)=-\frac{1}{2} \frac{(d-4) s}{(s+t) t} I_{I r r}^{d}(s, t) \\
& +\frac{1}{2} \frac{(d-4) s}{s+t} I_{1}^{d}(s, t) \\
& -9 \frac{(d-4)}{s t} I_{\text {Diag }}^{d}(s, t) \\
& +12 \frac{(d-3)}{(s+t) t} I_{B b o x}^{d}(s, t) \\
& +2 \frac{(d-3)^{2}(s+2 t)}{(d-4) s^{2} t(s+t)} I_{\text {Spec }}^{d}(s) \\
& +\frac{15}{2} \frac{(d-3)(3 d-10)}{(d-4) s t(s+t)} I_{B t r i}^{d}(s) \\
& +6 \frac{(d-3)(3 d-8)(3 d-10)}{(d-4)^{2} s^{2} t(s+t)} I_{\text {Sunset }}^{d}(s) \\
& +9 \frac{(d-3)(3 d-8)(3 d-10)}{(d-4)^{2} s t^{2}(s+t)} I_{\text {Sunset }}^{d}(t)
\end{aligned}
$$

Expanding eqs. (14,15) in $\epsilon$, and inserting the expansion of $I_{1}$ from ref. [ 3], of the pinched diagrams (6) (see, eg. [ 12]), and the result of eq. (13), we find that they are indeed satisfied.
Inspecting the right hand sides of the differential equations, one notices that in eq. (14), the coefficient of $I_{I r r}$, and in eq. (15), those of $I_{1}$ and $I_{\text {Irr }}$, are all proportional to $d-4$. This means that, if $I_{1}$ is known to $\mathcal{O}\left(\epsilon^{0}\right)$, the $\mathcal{O}\left(\epsilon^{0}\right)$ part of $I_{I r r}$ is, a priori, only determined by the system of equations up to a $t$-independent constant.

One also observes that the system of differential equations has a singular point at $s+t=0$. This corresponds to the special kinematical configuration where $p_{1}+p_{3}=0$. At this point, the numerator of $I_{I r r}$ becomes reducible:

$$
\begin{aligned}
P_{8} & =\left(k+p_{1}+p_{2}+p_{3}\right)^{2} \\
& =\left(k+p_{2}\right)^{2}=P_{1}-P_{2}+P_{3}-s,
\end{aligned}
$$

so $I_{\text {Irr }}$ collapses to a linear combination of $I_{1}$ and pinched diagrams.

For a further discussion of this singular point, we refer to the contribution of Gehrmann and Remiddi [ 13], who recently investigated the (equivalent) system of differential equations for $I_{1}$ and $I_{2}$. By excluding singular solutions of the differential equations as possible candidates, they were able to extract the order $\epsilon$ piece of $I_{1}-t I_{2}$ using only the value of $I_{1}$ at $s+t=0$ to $\mathcal{O}\left(\epsilon^{0}\right)$ as input. The information contained in this quantity is equivalent to our eq. 131).

\section{6 dimensions}

The master integrals $I_{1}$ and $I_{2}$ are both finite in $d=6$ dimensions [ 14]. This can be deduced from power counting considerations in momentum space; it is also easy to see by examining the arguments of the gamma functions in the Mellin-Barnes representation (11). Following the method of [ [ 4 ], we relate these master integrals in $d=6-2 \epsilon$ dimensions to master integrals of the new basis in $d=4-2 \epsilon$ dimensions. Substituting $\epsilon$-expansions for the latter, we find that all pole terms indeed cancel, and the finite parts are

$$
\begin{aligned}
& I_{1}^{d=6}=-\pi^{6}\left\{\frac{a_{1}}{(s+t)}+\frac{b}{t}\right\}, \\
& I_{2}^{d=6}=-\pi^{6}\left\{\frac{a_{2}}{s(s+t)}+\frac{b}{s t}\right\}, \\
& a_{1}=t \frac{\partial a_{2}}{\partial t}-6 \zeta(3),
\end{aligned}
$$




$$
\begin{aligned}
a_{2}= & \frac{\pi^{4}}{10}+6 \mathrm{Li}_{4}(-t / s)+\left(\ell^{2}+\pi^{2}\right) \operatorname{Li}_{2}(-t / s) \\
& +4 \ell\left(\zeta(3)-\mathrm{Li}_{3}(-t / s)\right)+\frac{\pi^{2}}{6} \ell^{2}, \quad(20) \\
b= & \left(2 \zeta(3)-2 \operatorname{Li}_{3}(-t / s)-\frac{\pi^{2}}{3} \ell\right) \log (1+t / s) \\
& +\frac{1}{2}\left(\ell^{2}+\pi^{2}\right) \log ^{2}(1+t / s) \\
& +\left(2 \ell \log (1+t / s)-\frac{\pi^{2}}{3}\right) \operatorname{Li}_{2}(-t / s) \\
& +2 \ell \mathrm{S}_{1,2}(-t / s)-2 \mathrm{~S}_{2,2}(-t / s) .
\end{aligned}
$$

We checked these expressions by numerical integration of Feynman parametric representations for the integrals in $d=6$ in the region of negative $s$ and $t$. This gives us an independent check on the finite parts of our result for $I_{I r r}$, eq. (13), and of $I_{1}$ in ref. [ [3].

\section{Conclusion}

We have resolved a technical issue related to the reduction of on-shell massless double box diagrams to master integrals. Looking back, one can say that the basis of master integrals $\left\{I_{1}, I_{2}\right\}$ used in ref. [ [1] was an unfortunate choice for applications in $d=4-2 \epsilon$ dimensions, because it leads to factors of $1 / \epsilon$ in the reduction coefficients. The troublesome terms do not cancel in physical amplitudes [ 2]. If one uses, instead, the basis $\left\{I_{1}, I_{I r r}\right\}$, the factors of $1 / \epsilon$ disappear. Our main result is the expansion of the integral $I_{I r r}$ in terms of polylogarithms to $\mathcal{O}\left(\epsilon^{0}\right)$, eq. (13), which we derived from scratch using a Mellin-Barnes representation. (An equivalent result was obtained independently by Gehrmann and Remiddi [13, by solving a system of differential equations and using the first master integral from ref. [3] as input). We verified that our result, together with the first master integral, satisfies the differential equations (14 15). As a further check on the constant which is not determined by the differential equations alone, we used our result to derive analytical expressions for two finite double box integrals in $d=6$ dimensions, eqs. (17,18), and checked them by numerical integration.

\section{Acknowledgements}

We thank E.W.N. Glover, D.J. Broadhurst and O.L. Veretin for discussions and useful suggestions. C.A. acknowledges financial support from the Greek Government, J.B.T. acknowledges financial support from the DFGForschergruppe "Quantenfeldtheorie, Computeralgebra und Monte-Carlo-Simulation", and M.E.T. acknowledges financial support from CONACyT and the CVCP. We gratefully acknowledge the support of the British Council and German Academic Exchange Service under ARC project 1050 .

\section{REFERENCES}

1. Z. Bern, L. Dixon and D. A. Kosower, JHEP 0001 (2000) 027 hep-ph/0001001.

2. E.W.N. Glover and M.E. Tejeda-Yeomans, these proceedings.

3. V. A. Smirnov, Phys. Lett. B460 (1999) 397 hep-ph/9905323.

4. V. A. Smirnov and O. L. Veretin, Nucl. Phys. B566 (2000) 469 [hep-ph/9907385.

5. O. V. Tarasov, Phys. Rev. D54 (1996) 6479 hep-th/9606018; O. V. Tarasov, Nucl. Phys. B502 (1997) 455 hep-ph/9703319.

6. F. V. Tkachov, Phys. Lett. B100 (1981) 65; K. G. Chetyrkin and F. V. Tkachov, Nucl. Phys. B192 (1981) 159.

7. T. Binoth and G. Heinrich, hep-ph/0004013.

8. N.I. Ussyukina, preprint FERMILAB-PUB93/241-T; N.I. Ussyukina, hep-ph/9801261.

9. N. I. Ussyukina and A. I. Davydychev, Phys. Lett. B298 (1993) 363.

10. L.J. Slater, Generalized Hypergeometric Functions, Cambridge University Press, 1966.

11. T. Gehrmann and E. Remiddi, hep$\mathrm{ph} / 9912329$.

12. C. Anastasiou, E.W.N. Glover and C. Oleari, Nucl. Phys. B565 (2000) 445 hepph/9907523]; C. Anastasiou, E.W.N. Glover and C. Oleari, hep-ph/9912251.

13. T. Gehrmann and E. Remiddi, these proceedings hep-ph/0005232.

14. Z. Bern et al., Nucl. Phys. B530 (1998) 401. 